\title{
ABSORPTION OF SHORT-PULSE ELECTROMAGNETIC ENERGY BY A RESISTIVELY LOADED STRAIGHT WIRE.
}

E. R. Miller, F. J. Deadrick, and J. A. Landt

October 17, 1975

\section{MASTER}

Prepared for U.S. Energy Researc 7 \& Deyelopment Administration under contract No. W-7405.Eng-48

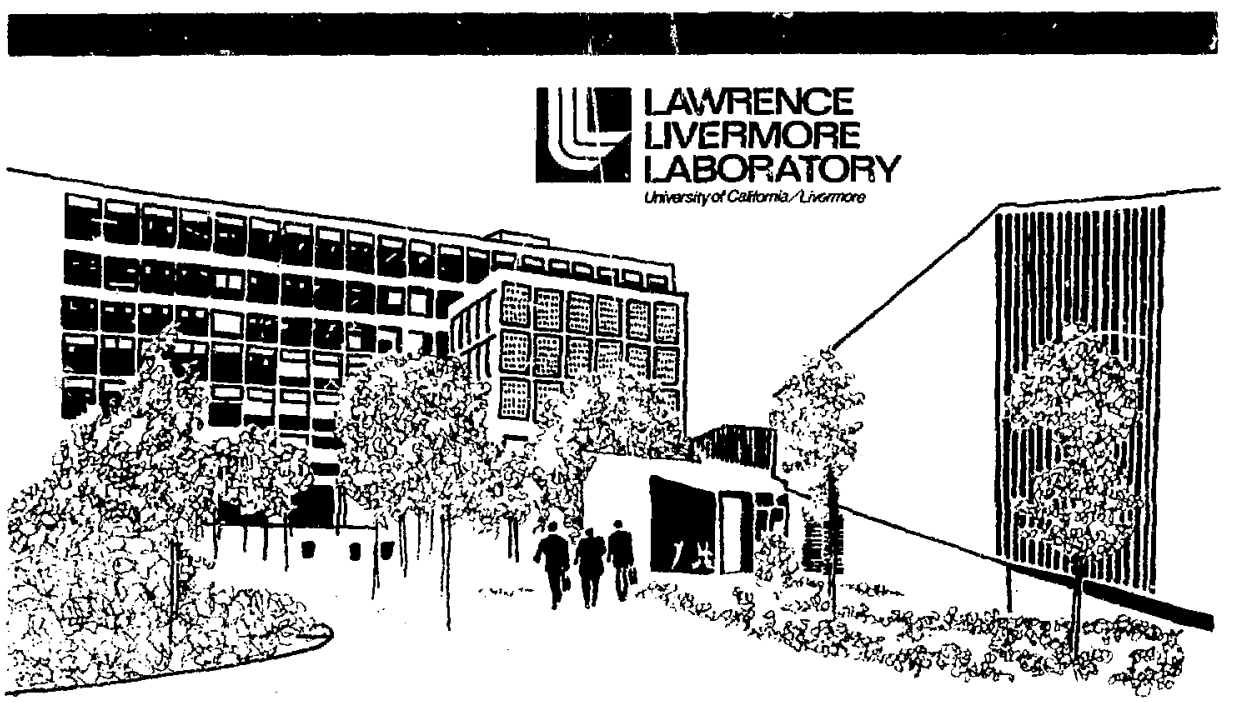

$1+\cdots+\cdots$ 
"This report was prepared as en eccount of work sponsored by the Itnited States Government. Neither the United States nor the United States Energy Research \& Development Administration, nor any of their employees, nor eny of their contructors, subcontraciors, or their employees, makes any warrenty, express or implied, or assumes any lege? whrenty, express or implied, or assumes any lege
liability or responsibility for the accuracy, compiteteness or usefulness of any information, apparatus, product or process disclosed or represents that its use would not infringe prtvately-owned rights."

Printed in the United States of America Available from

National Technical Iriormation Service

U.S. Departmer'ic of Commerce 5285 Port Royal Road Springfield, Virginia 22151

Price: Printed Copy $\$$ *; Microfiche $\$ 2.25$

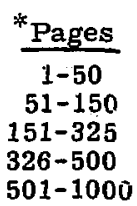

NTIS

Selling Price

$\$ 4.00$

$\$ 5.45$

$\$ 7.60$

$\$ 10.60$

$\$ 13.60$ 


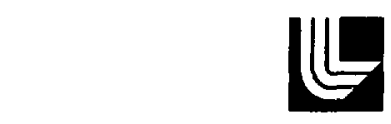

LAWRENCE LJVERMORE LABORATORY

University of Ci fornia/Livermore, Calitomia 94550

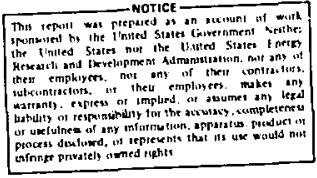

UCRL-51936

\section{ABSORPTION OF SHORT-PULSE ELECTROMAGNETIC ENERGY BY A RESISTIVELY LOADED STRAIGHT WIRE}

E. K. Miller, F. J. Deadrick and J. A. Landt*

MS. date: October 17, 1975

*Los Alamos Scientific Laboratory, P.o. Box 1663, Los Alanos, NM 87545 


\title{
ABSORPTION OF SHORT-PULSE ELECTROMAGNETIC ENERGY \\ BY A RESISTIVELY LOADED STRAIGHT WIRE
}

\begin{abstract}
Here, absorption of short-pulse electromanetic energy by a resistively loaded straight wire is examined. Energy collected by the wire, load energy, peak load currents, and peak load voltages are found for a

wide range of parameters, with particular emphasis on nuclear electromagnetic pulse (EMP) phenomena. A series of time-sequenced plots is used to illustrate pulse propagation on wires when loads and wire ends are encountered.
\end{abstract}

\section{Introduction}

The short-pulse response of objects illuminated by electromagnetic radiation is of interest in such problem a:eas as nuclear electromagnetic pulse (tikP) and electromagnetic vulnerability (EMV). Of particular concern is the energy that may be delivered to a load connected to or contained within the illumi.nated object due to the energy collected by it from the incident pulse.
Because the interaction can occur over a broad frequency band, the familiar narrow band, or monochromatic view point, may not be appropriate for understanding of the transient phenomena that take place. For this reason, we are studying the problem using a direct time-domain-solution procedure. This report describes our approach and summarizes the results obtained to date.

\section{Numerical Approach}

Since we are interested in 1 inear loads, the analysis can be performed in either the time or frequency domain. However, the computational efficiency of the time domain is sig- nificantly greater than of the frequency domain for the kind of problem considered here (i.e., determining the wide-band response of an ooject to one or more incident fields). 
Therefore, the results which follow were obtained using a time-dependent thin-wire integral equation based on the electric field. Detalls of tne moment-method freatment, which incorporates a ninc-paint LaGrangian current basis in space-time together with delta-function weights and the time-stepping solution of an initial value problem, can be found in references 1 and 2 .

For a given transient excitation, which either can be localized to study an antenna problem or spread over the entire object to analyze a scattering problem, the induced current and charge are found as a function of their space-tine behavior. From such results, we are able to derive peak load-current $\left(I_{L}\right)$ and voltage--jrop $\left(v_{L}\right)$ values as well as the instantaneous power dissipated by the load $\left(P_{L}\right)$ and collected by the object $\left(P_{C}\right)$ and cumulative energy dissipated in the load $\left(E_{L}\right)$ and collected by the object $\left(E_{C}\right)$. These quantities are defined as follows:

$$
\begin{aligned}
I_{L}= & \max \left|I\left(s_{L}, t\right)\right| \\
V_{L}= & \max \mid I\left(s_{L}, t\right) R_{L} \\
& +r_{L} \frac{d}{d t} I_{L}\left(s_{L}, t\right) \\
& -\left.\frac{1}{C_{L}} \int_{-\infty}^{t} \frac{d}{d s} I_{L}(s, \tau)\right|_{s=s_{L}} d \tau \mid \\
P_{L}(t) & =I^{2}\left(s_{L}, I\right) R_{L}
\end{aligned}
$$

$$
\begin{aligned}
& P_{C}(t)=\int_{C(\bar{r})} E^{I N C}(s, t) \cdot S I(s, t) d s \\
& E_{L}(t)=\int_{-\infty}^{t} P_{L}(i) d t \\
& E_{C}(t)=\int_{-\infty}^{t} P_{C}(t) d t
\end{aligned}
$$

where the integrals and differentials in the above equations are approximated in terms of the numerically derfved space-time current values; $I(s, t)$ is the current at location $s$ and time $t ; \bar{E}^{\mathrm{INC}}$ is the incident electric field; and $c(\bar{r})$ is the object's contour. In addition, the presence of a single, lumped load is assumed, a]chough the analysis permits distributed loads to be pandled with equal facility. Results presented in the following paragraphs are primarily for $E_{L}$ and $F_{C}$. The numerical calculations geneiclly were performed for a nominal EMP two-term exponential pulse given by

$$
\begin{aligned}
\bar{E}^{I N C}(t)= & 5.25 \times 10^{4}\left[\exp \left(4.0 \times 10^{6} t\right)\right. \\
& \left.-\exp \left(4.7 \times 10^{8} t\right)\right] .
\end{aligned}
$$

The test object in this study was a straight wire of length $L$ and radius a. The wire was modeled using 30 segments of length $\Delta=\mathrm{L} / 30$ and $a$ time step $\delta=\Delta i \therefore$. This means computed results should be valid to a maximum $L / \lambda$ value of approximately 
5. The quantitative impact of the

high-f requency cutof $f$ in computational

accuracy on the results obtained is negligible for wires less than

$\checkmark 1,000 \mathrm{~m}$ long because of high-

frequency fallof $f$ in the EMP spectrum (discussed later).

For the nominal configuration, we examined a pulse at broadside incidence on a wire of variable length with $\mathrm{L} / \mathrm{a}=$ constant $=148.4(\Omega=10)$ and lump loaded at its center (load confined to the two center segments) by a resistance of variable value. Variations in incidence angle, ioad posttion, loadphase angle, and $L / a$ value were also studied. The results of these studfes are summarized in the next section.

\section{Numerical Results}

Figure la shows the load current and voltage axcited on a 10-m-long wire for the nominal case with the load resistance as a parameter. Note that the current appears to be critically damped for $Q_{L}=1 \mathrm{~K}$ ?, under damped for $R_{1}=1:$, and overdamped for $R_{l}=1 \mathrm{~N}$. Also shown (Figs. $1 \mathrm{~b}$ through le) are the ins cantaneous collected power and load power and the cummulative collected energy and load energy. Observe that, while load power must always be positive (leading to a monotonically increasing load energy), collected power may be negatfve. This indicates that the rate of energy re-radiation can at times exceed the rate of energy collection.

These results, typtcal of the current waveforms observed $f$ a wide range of wire lengths, essentially characterize step-function illumination because of the short rise time and long decay time of the ERP. All

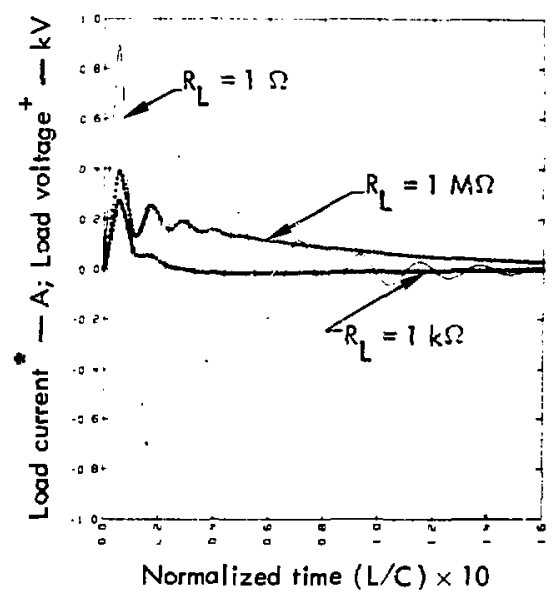

Applies to $R_{L}=1 \Omega$ and $R_{L}=1 \mathrm{k} \Omega$ only. tApplies to $R_{L}=1 \mathrm{k} \Omega$ and $R_{L}=1 \mathrm{M} \Omega$ anly.

Fig. Ia. Load currents and voltages shown as a function of time for a 10-m dipole illuminated by a broadside incident LMP. Three load values shown correspond to under damped, critica1ly damped, and over damped conditions. 
remaining data presented in this report was derived from calculations and results similar to those in Figs. la through le and dif ined by Eqs. (1-6).

The energy collected from the incident pulse by the wire, $E_{C}$, and the energy delivered to the load, $E_{L}$, are shown as a function of load resistance for wire lengths of 1,10 , 100 , and $1,000 \mathrm{~m}$ in Figs. $2 a$ and $2 b$. It can be seen that the loald energy approaches within $10 \%$ of the collected energy tor a broad range of resist-

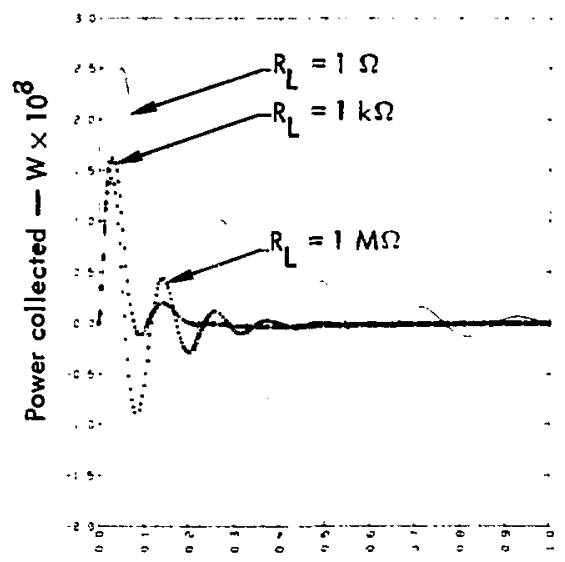

Normolized time $(\mathrm{L} / \mathrm{C}) \times 10$

Fig. 1b. Instantaneous power collected by a 10-m dipole 1lluminated broadside by an incident EMP wave for three values of load reststance. Note, negative energy collection indicates that the rate of re-radiation exceeds che rate of energy collection.

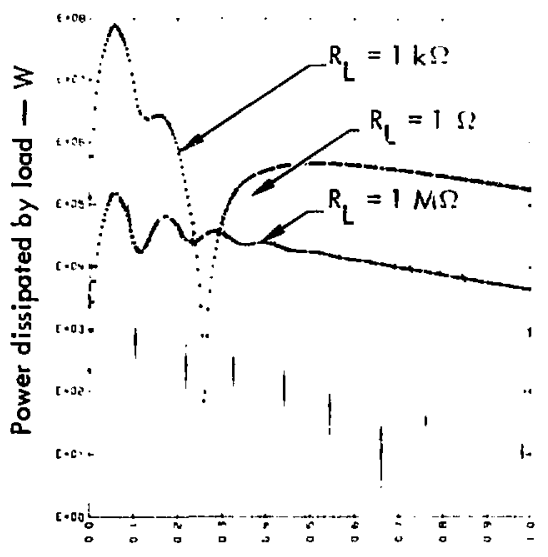

Normalized rime $(L / C) \times 10$

Fig. 1c. Power disslpated in under damped, crltically damped, and over damped loads, as a function of time for the incitent E!P wave.

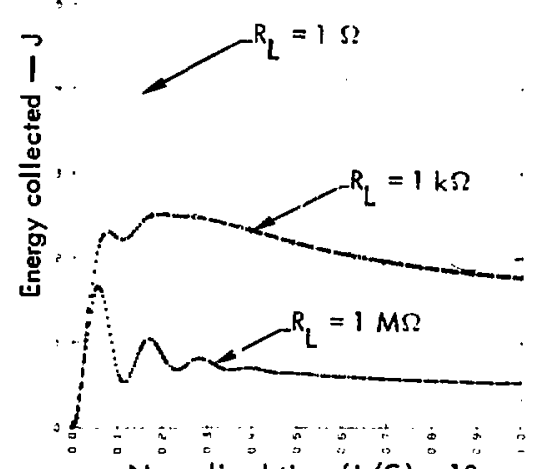

Normolized time $(\mathrm{L} / \mathrm{C}) \times 1 \mathrm{C}$

Fig. 1d. Cuminulative collected energy for a 10-n dipole flluminated by an EMP. 


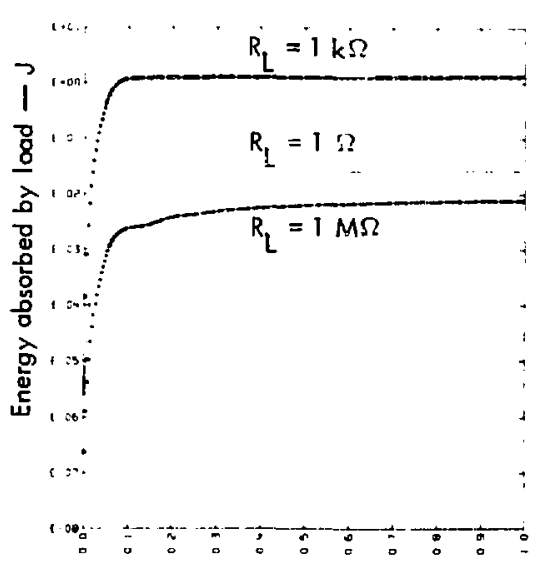

Normalized time (L/C) $\times 10$

Fig. le. Energy absorbed by the loac values of $1,1 \mathrm{~K}$, and $1 \mathrm{M}$ ohms. Note that the case of critical damping ( $1 \mathrm{~K}$ ohms) corresponds to the maximum energy absorption.

ance values (100 to $1,000 \Omega$ ). This result is significantly different from that for monochromatic excitation, both in tarms of the fraztion of collected energy delivered to the loud and in terms of the load value that maximizes the load energy. This difference is probably due to the distinctly different reradiation characteristics of wires under narrowversus wide-band electromagnetic illupintion.

Based on the results of calculations like those from which the data in Figs. 2a and b were derived, it is eas) to determine load response as a function of load and length parameter variations. Plots depicting contours of constant energy, current, etc. are shown in Figs. 3a through $3 \mathrm{~d}$ as a function of wire length and load resistance. These curves were obtained from time-domain calculations for wire lengths of $1,10,100$, and $1,000 \mathrm{~m}$ and for load values of 1,10 , $\ldots, 10^{6}$ ?. They could have also been developed from transferadmittance and input-impedance calculations for a single wire length and load combination. $j$ This kind of data (Figs. 3a through 3d) is quite useful in EMP applications for performing "quick-look" assessments of induced threas. As shown In Figs. $2 a$ and $2 b$, $a$ purely resistive load can absorb nearly all the energy collected by a straight wire under EST illumination. A complex load could iurther increase that portion of collected energy disstpated in the load. Figure 4 illustrates this point, showing contours of constant load energy as a function of load resistance and reactance. The latter is a simple inductance or capacitance, with the reactance value referred to frequency where $L / \lambda=1 / 2$. A slight increase in energy absorption by a tomplex load is found to occur relative to the case of a purely reststive load. The change, however, is orly $n 10 \%$ or less. This also agrees with results obtained by Merewether. 4 


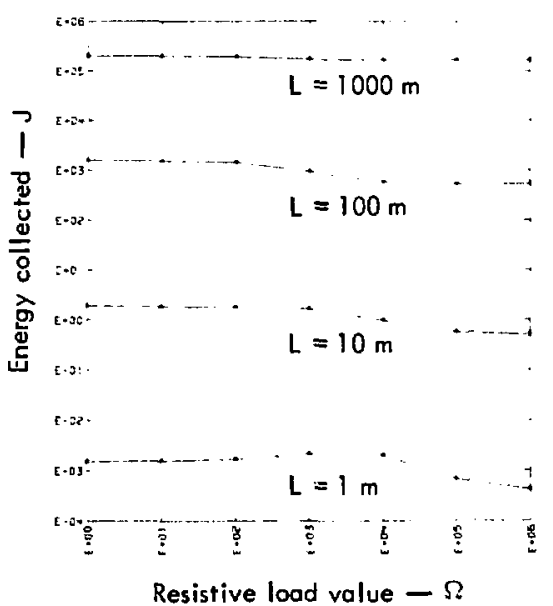

Fig. 2a. Energy collected versus resistive load for dipoles $1,10,100$, and $1000 \mathrm{~m}$ in length. Results shown are for center-loaded dipoles illuminated by EMP waves at broadside incidence. Curves indicate that load resistance has little effect on the energy collected by the structure.

Finally, Figs. 5a through $5 i$ show che effects of varying load position, angle of incidence, and $\mathrm{L} / \mathrm{a}$ value for a wire $10 \mathrm{~m}$ long. Concerning the position and angle-of-

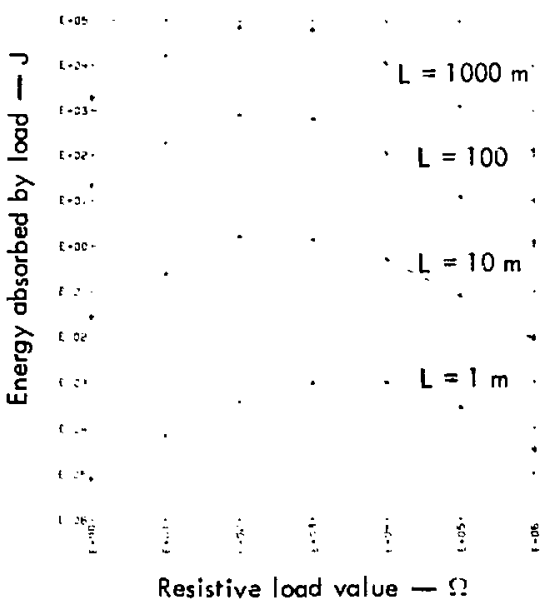

Fig. 2b. Curves show energy absorbed by loads. In each case, energy absorption was maximized at a critical load value. Energy absorbed by the load is less than the energy collected by the dipole.

incidence variations, we find that collected and absorbed energy are maximum for the midpoint load and broadside illumination. Furthermore, increasing wire radius increases both coilected and absorbed energy (as expected from the scattering properties of dipoles). 

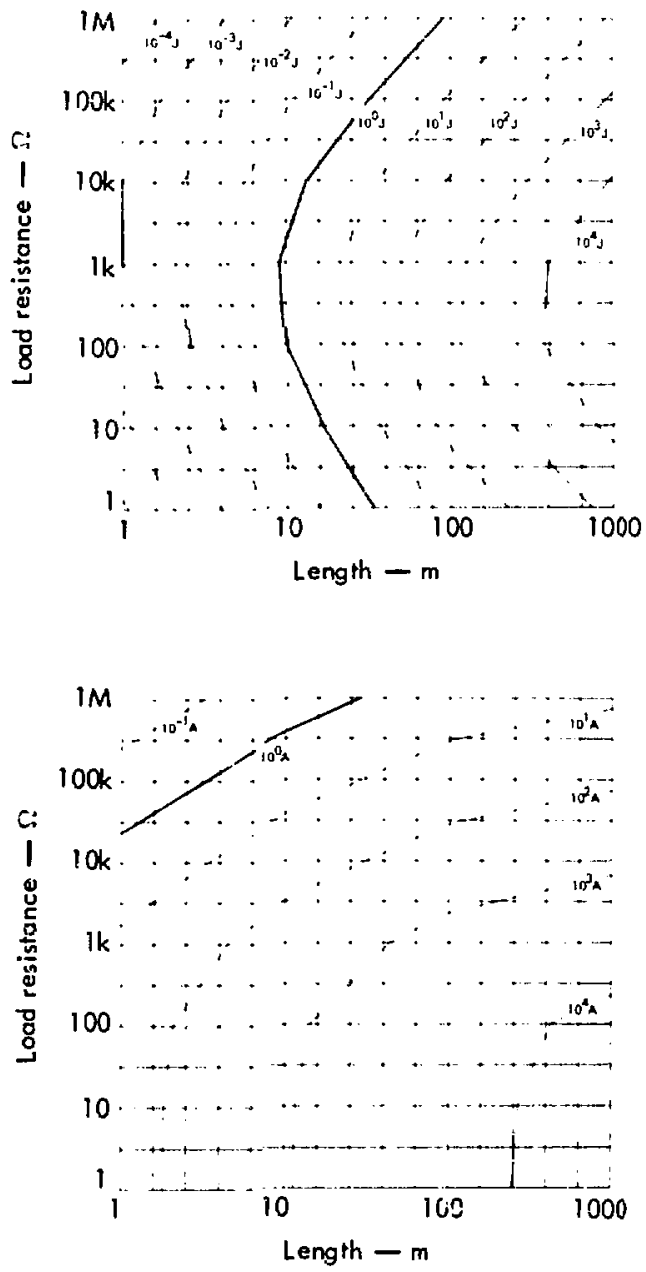

Fig. 3a. Contours show peak load energy as a function of dipole lel.gth and load resistance. Again, results are for a dipole illuminated by a broadside incident EMP wave.
Fig. 3b. Contours show peak load current for paramecers of wire length and load resistance value. 


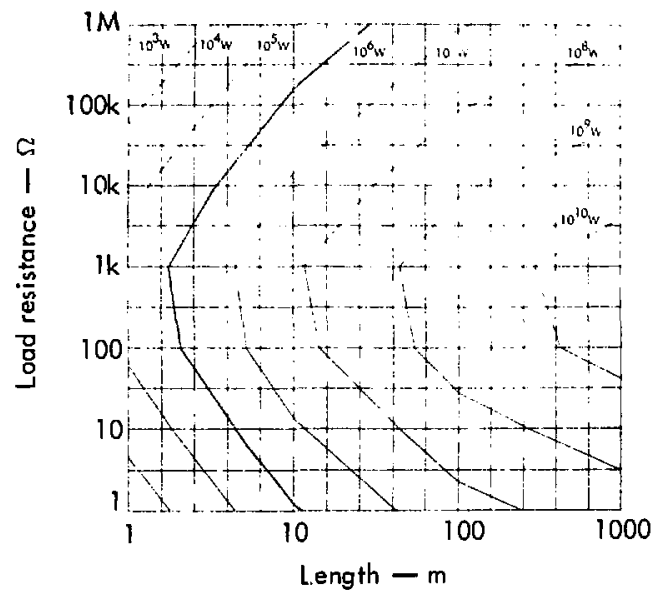
Fig. 3c. Contours show peak load power dissipa- tion as a function of load value and dipole length.

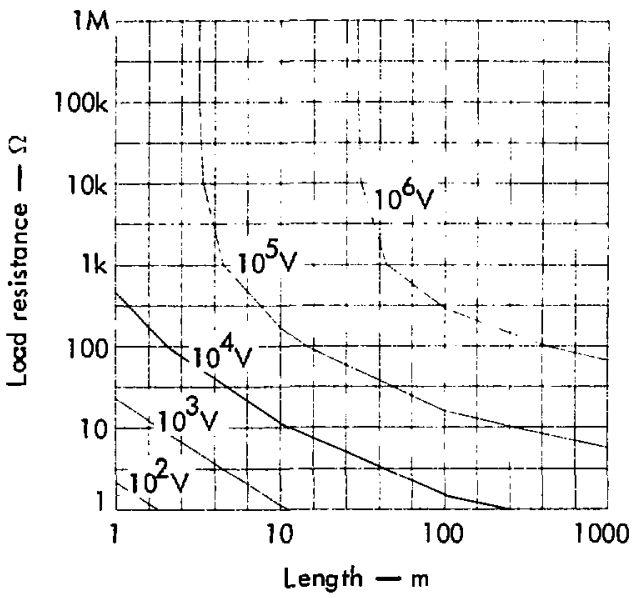

Fig. 3d. Contours show peak load voltage as a function of dipole length and load resistance. Contours correspond to a dipole illuminated by a broadside incident EMP wave. 


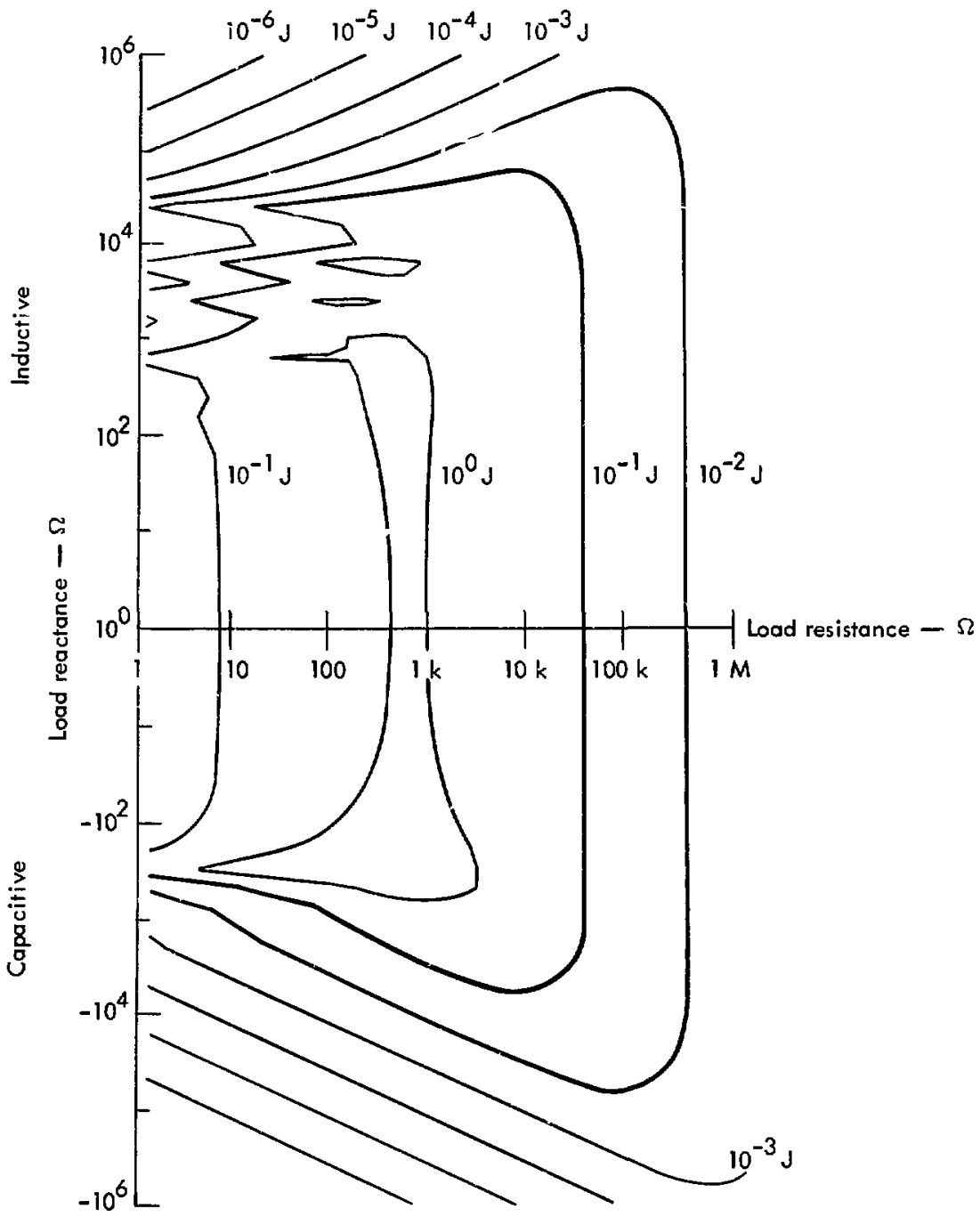

Fig. 4. Contours show load energy as a function of load resistance for a $10-m$ dipole illuminated by an EMP at broadside incid :e. 


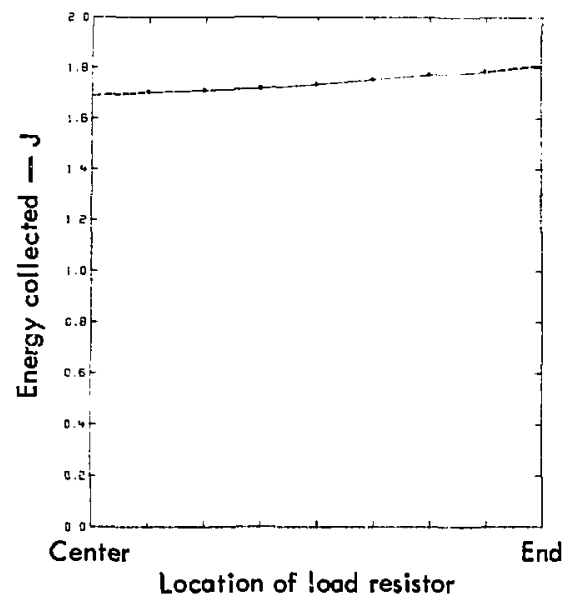

Fig. 5a. Energy collected by a 10-m dipole as a function of load position. Results shown are tor half of the dipole, with load position being varied from its center outward to its end.

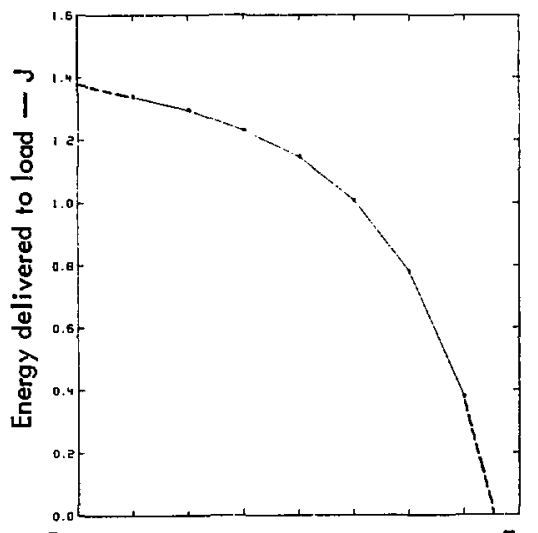

Center End Location of load resistor

Fig. 5b. Energy dissipated by load as $z$ function of load position.

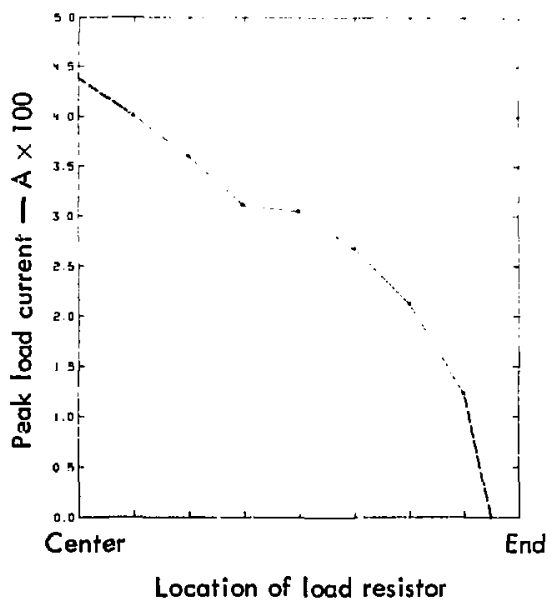

F1g. 5c. Peak load current as a function of load position for a 10-m dipole illuminated at broudside by an Incident EMP wave.

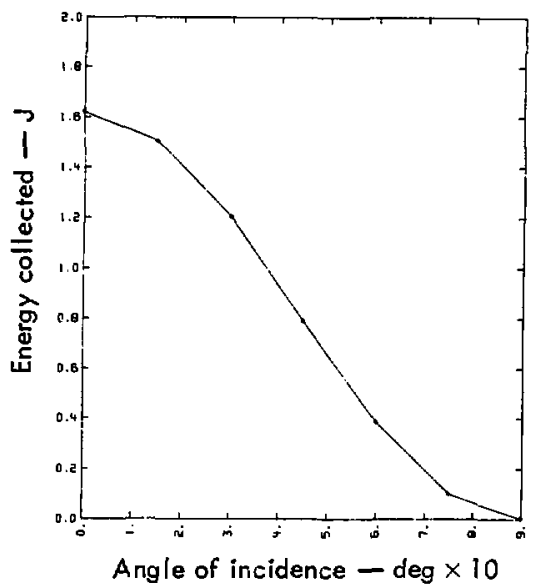

Fig. 5d. Energy collected by a 10-m dipole with a $500-\Omega$ load as a function of the angle of incidence of the incident EMP wave. 


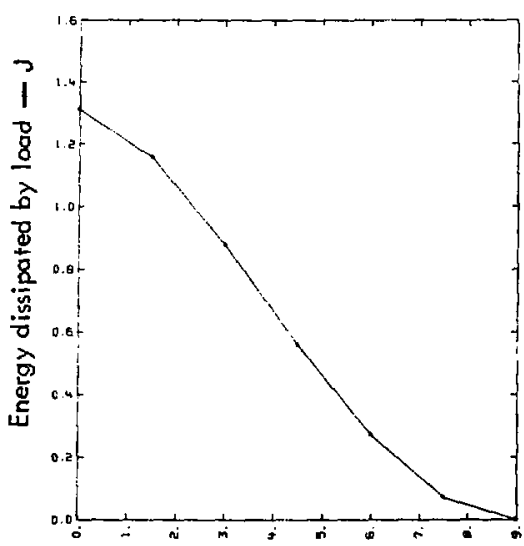

Angle of incidence $-\operatorname{deg} \times 10$

Fig. 5e. Energy dissipated by a $500-\Omega$ load at the center of a 10-m dipole as a function of the angle of incidence of the incident EMP wave.

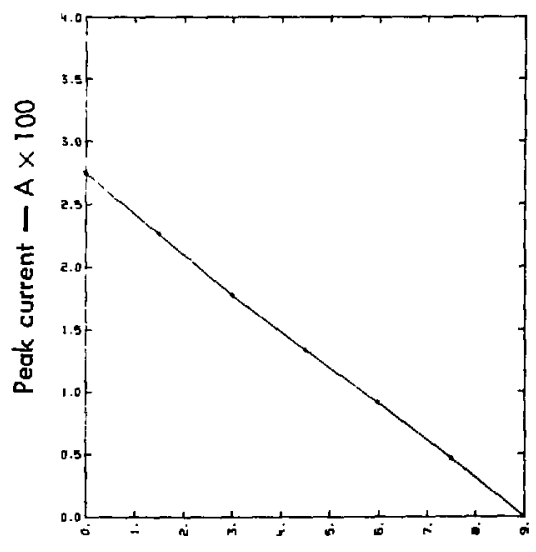

Angle of incidence $-\operatorname{deg} \times 10$

Fig. 5f. Peak load current as a function of angle of incidence of the incident field.

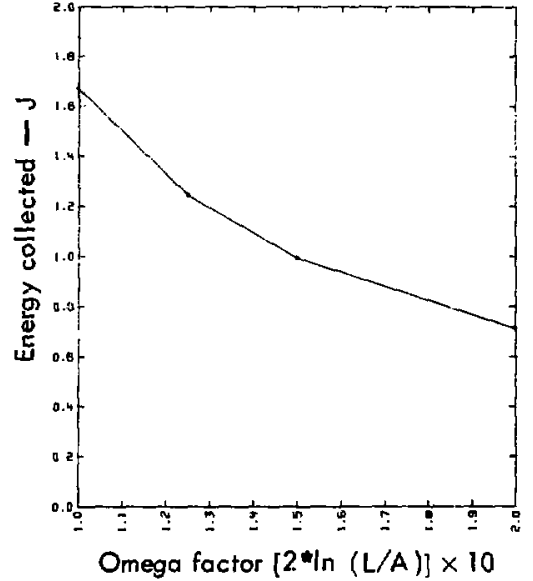

Fig. $5 \mathrm{~g}$. Energy collected by a 10-m dipole as a function of the thickness of the dipole wire. Here, wire thickness is varied from a factor of $\mathrm{L} / \mathrm{a}$ of 148 to an L/a of $2.2 \times 10^{4}$.

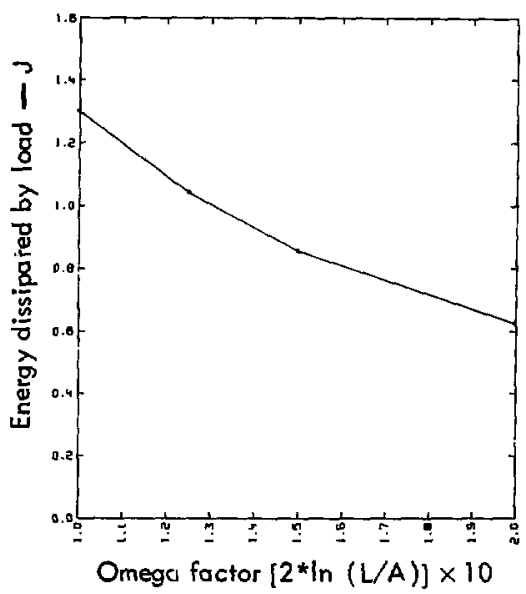

Fig. 5h. Energy disslpated in a $500-\Omega$ load as a function of dipole thickness. 


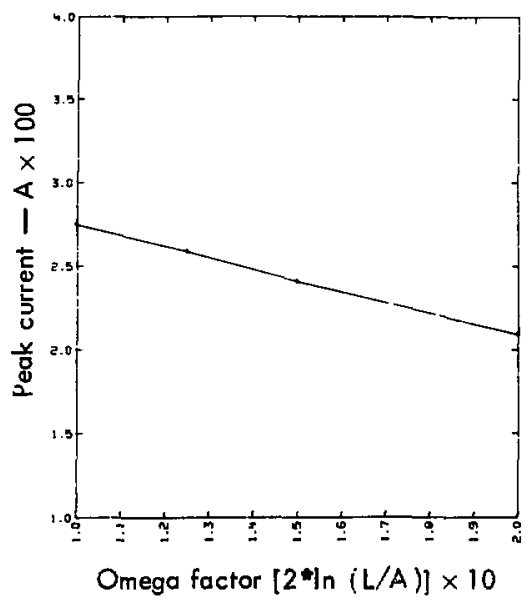

Fig. 5i. Peak current through a $500-?$ load as a function of dipole thickness.

\section{Discussion of Results}

The most unexpected finding in this study was that more than half of the energy collected by an EMPilluminated object can be delivered to the load. This result is certainly different from the more familiar monochromatic case, where maximum load power is half that collected under conjugate-matched-load conditions. The behavior observed for pulse excitation can be explained qualitatively as follows.

Consider a center-loaded straight wire excited by an EMP at broadside incidence. Numerically, the wire can be regarded as an $\mathrm{N}$-port network, where $\mathbf{N}$ is the number of segments used to model the wire. Thus, total wire current can be derived by super- positioning the individual contributions of the $N$ ports, and overall current behavior can be inferred from the current of a representative port. The current induced at a given port on the wire scatterer is identical to that which results when the wire is excited at the port as an antenna by the same tangential electric field. The current pulse induced on the antenna at the driven port divides into two oppositely propagating waves of equal amplitude. Initially, almost al1 of the energy supplied by the source, or generator (given by $\Delta S E I d t)$, is contained in the current. But, as the original current pulse divides into two separate fulses propagating in opposite directions, 
more of the energy is stored in the tharge. Eventually, a steady-state condition is reached, in whici onefourth of the generator-supplied energy is in each of the oppositely propagating current and charge pulses. This sequence of events is shown in Fig. 6.

What happens when one of the current-charge pulses encounters a resistive load is shown in FIg. 7 . For a load of $600 \Omega$, the transmitted and reflected pulses are approximately half the amplitudes of the Incident pulse. Consequently, half of the incident energy must have been absorbed by the load (as is verifted by computing $E_{L}$ ).

Figure 8 shows the reflection of a current-charge pulse fiom the open end of a wire. In this case, about $60 \%$ of the energy contained in the pulse incident on the open end of the wire appears in the reflerted pulse, the rest having been radiated upon reflection.

Both events described above (Figs. 7 and 8 ) provide insıght into the behavior of the pulse-excited scatterer. The discussion would be incomplete, however, without considering the situation in which two ports located symmetrically about the load are excited simultaneously. The out-

\footnotetext{
*As obtained by integrating the square of the current and charge pulses before and after reflection.
}

come of such an event can be inferred from the results shown in Fig. 7 . But, to more clearly illustrate the phenomenon involved, we will treat this case explicitly, as shown in Fig. 9, again using a $600-2$ resistance load.

The current-charge pulse behavior in Fig. 9 is distinctly different from that in Figs. 7 in that there is essentially no reflected or transmitted current-charge pulse. This is caused by the mutual charge cancellation of the two pulses as they interact with the load. All incident energy is absorbed by the load and, thus, appear matched. Note, however, for a matched condition to be achieved, not only is the load value important; two waves having oppositely signed charges also are required because of charge conservation.

Considering the results shown in Figs. 7 through 9, the explanation for the energy-absorption characteristics found In the "Numerical Kesults" section becomes obvious. Essentially, all energy collected by the wire that results in current-charge pulses propagating towards the luad can be absorbed, while about 60 to $80 \%$ * of that which appears in pulses initially propagating towards the wire ands is also absorbed after one end reflection. 


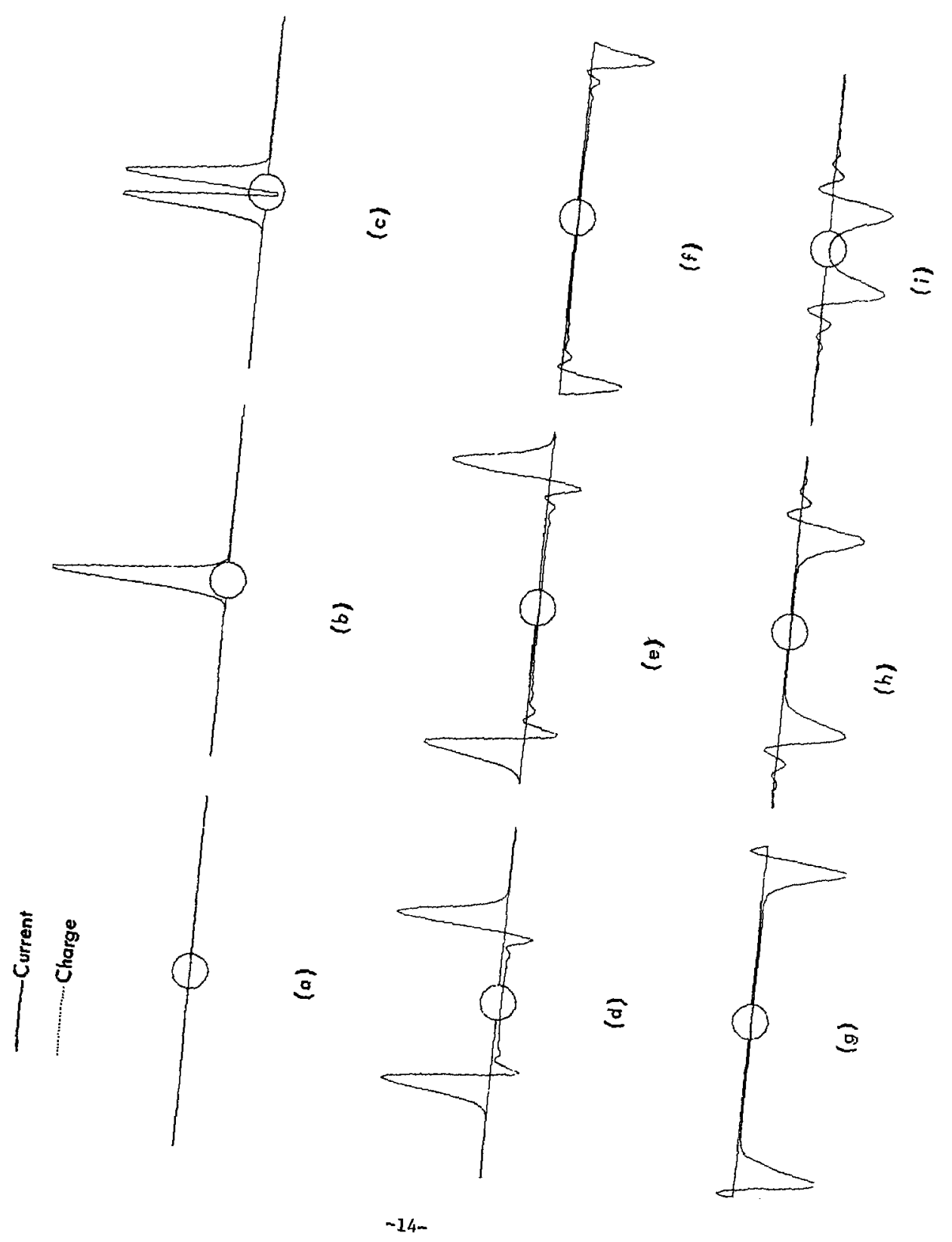


Fig. 6. Graphs show propagation of current and charge pulses on a center-excited dipole. In this case, the dipole is unloaded and excited at the center by a narrow gassian-shaped pulse. (a) Dipole

initially unexcited. (b) Gaussian pulse applied to dipole. (c) Pulse begins to divide with one current wave traveling to the right and the other to the left. ( $d$ and e) Current and charge pulses (solid and dotted lines respectively) propagate outward from the source. (f) Pulses reach end of dipole wires. When wire ends are reached, the charge shows a rapid buildup while the current pulse changes sign as it is reflected from the open end of the wire. Remaining parts of $f$ igure ( $g-1)$ show the reflected pulses now propagating inward toward the center of dipole. 

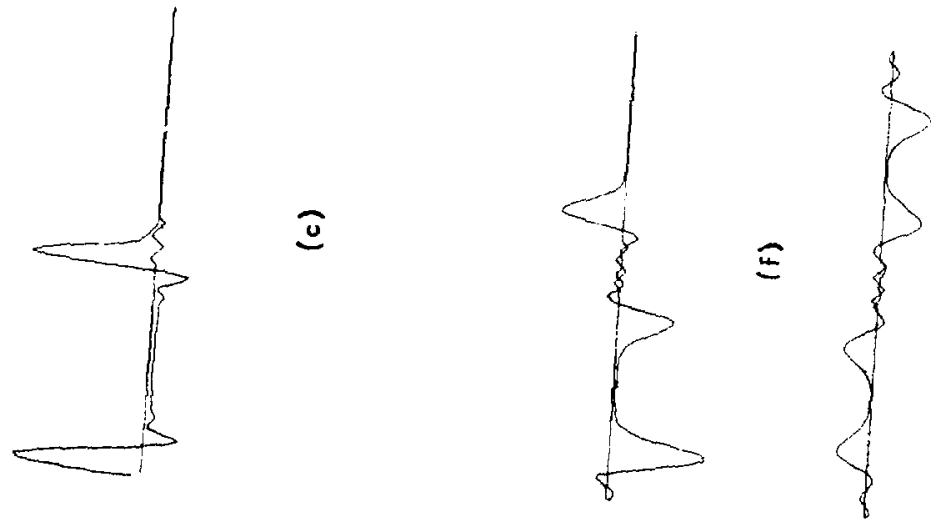

$=$

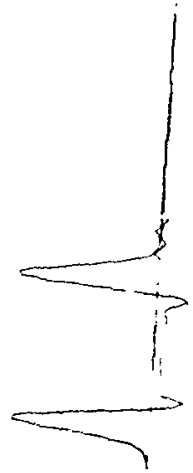

0
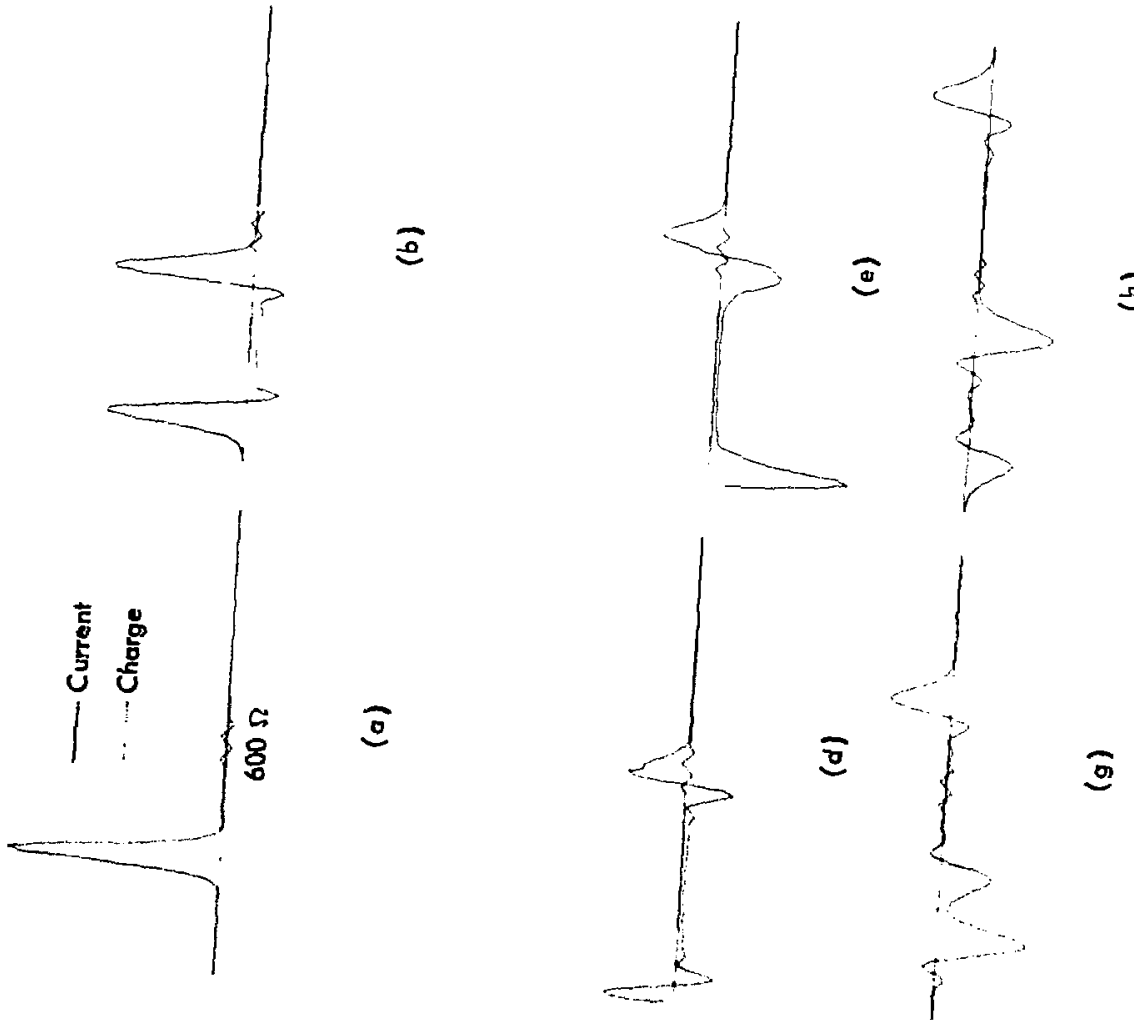
Flg. 7. Figure 1llustrates effect of load resistor on propagating current and charge pulses. In part (a), the antenna is excited by a narrow gaussian pulse and a $600-\Omega$ load is placed at the dipole center. At times (b) and (c) the pulse has split into two parts and has propagated outward to the load and the left end of the dipole. In (d), there is a charge buildup at the load and a decrease in the amplitude of the current pulse. Parts (e) and $(f)$ show the pulse at the load again spliting into two paris, half passing through the load to the rlght and half being reflected back to the left. The remaining frames [ $(g)$ through $(i)]$ show how the reflected pulses interact at later times. 

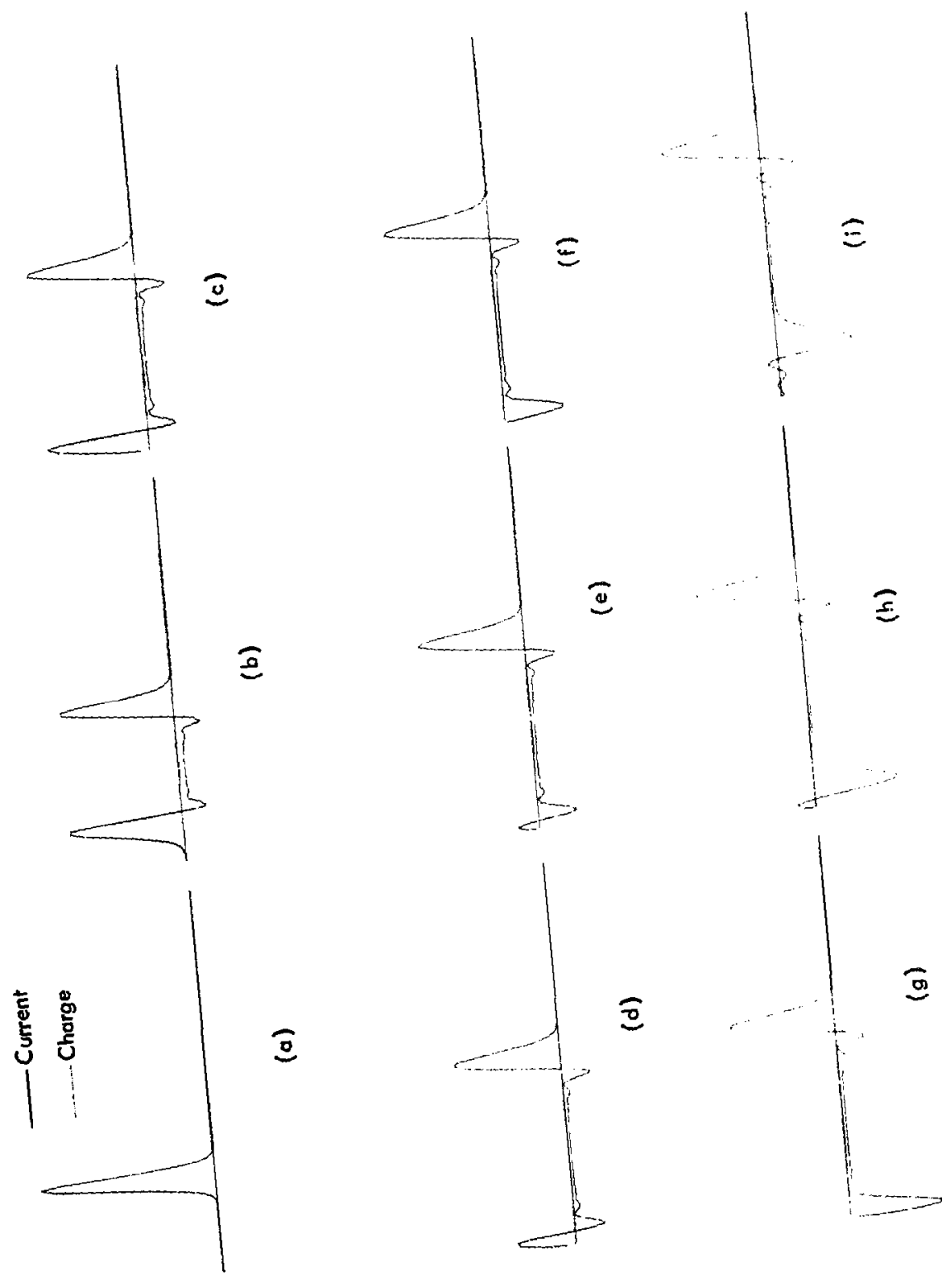

$-18-$ 
Fig. 8. Pulse refiection from the dipole end is shown here. As in the previous figures, the dipole is excited by a short pulse, which separates into left and right propagating pulses. When the leftward moving pulse reaches the wire end, we ses a very rapid buildup of charge at the wire end point. Then, upon seeing an open circuit, the current pulse reverses polarity and reflects back to the right. Note that the charge pulse does not reverse polarity upon reflection, due to charge conservation. 

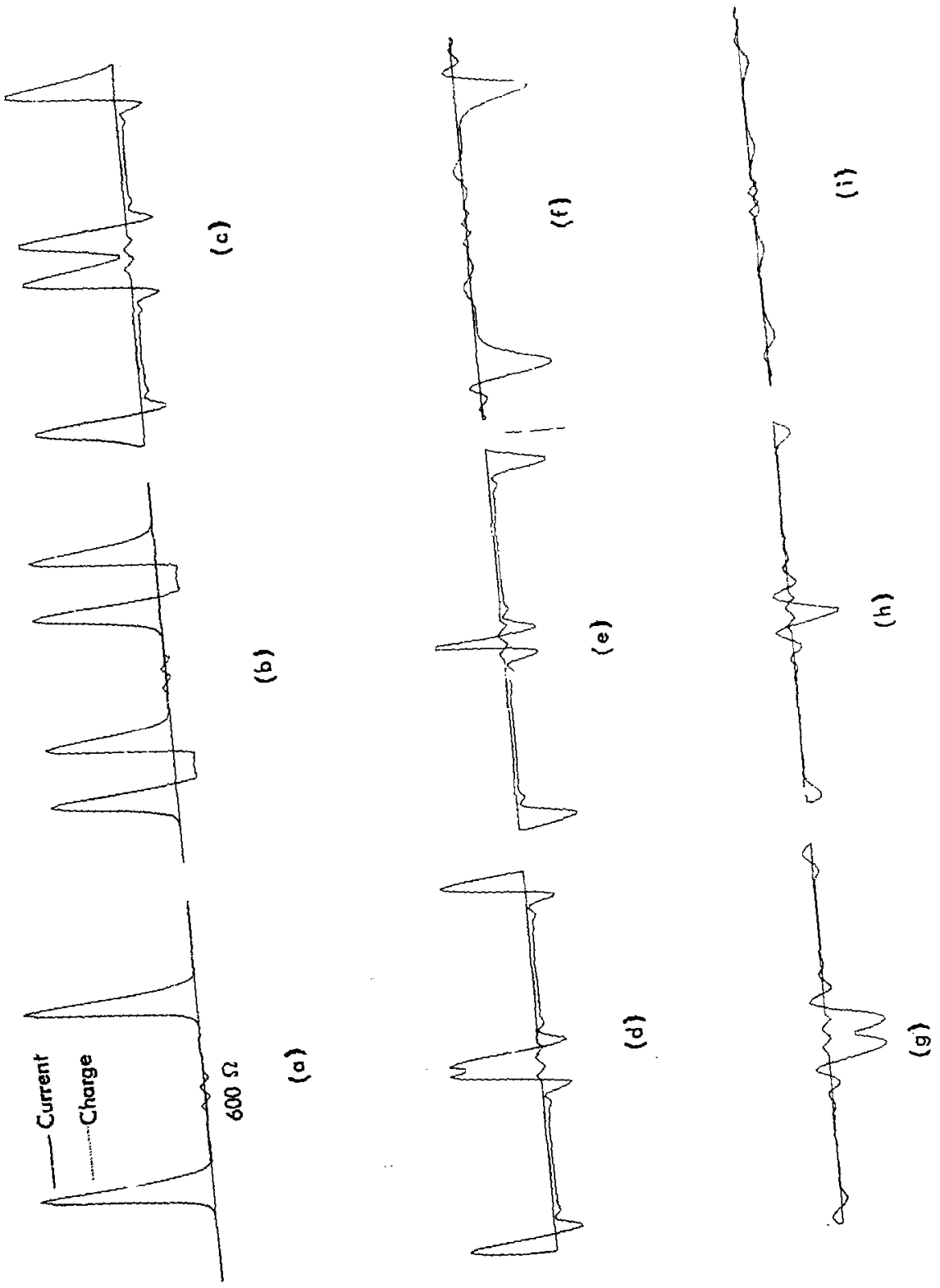

$-20-$ 
Fig. 9. Here, two symetrically located pulses are induced on the antula and allowed to propagate as shown. Note that the two current pulses propagating toward the load at the centir have the same polarity, while the charge pulses have opposite polarity. When the pulses reach tile load, a charge cancellation process occurs and the current pulses vanish. At time (g), the other two pulses have reflected from the end of the wire and have reached the load, and agotn charge canceliation occurs and the pulses disappei. 
Further study of other impedance discontinuities (e.g., wire bends, curvatures, and junctions) are planned and should provide additional information on the pulsed behavicr of antennas and scatterers.

\section{References}

1. A. J. Poggio, E. K. Milier, and

D. J. 'surke, J. Comput. Phys., 12, 210 (1973).

2. J. A. Landt, E. K. Miller, and

M. VanBlaricum, WT-MBA/LLLIB: A

Computer Program for the Time-

Doma1n Electromagnetic Response

of Thin-Wire Structures, Lawrence

Livermore Laboratory, Rept.

UCRL-5I585 (1974).
3. F. J. Deadrick, E. K. Miller, and

H. G. Hudson, The LLL Transient

Electromagnetics Measurement

Facility, Lawrence Livermore

Laboratory, Rept. UCRL-51933

(1975).

4. D. E. Mereweti:er, IEEE Trans.

Electromagnetic Compatab111ty

EMC-13 (No. 2), 41-44

(1971).

\section{Acknowledgments}

The authors wish to acknowledge the Defense Nuclear Agency (Subtask

Code R99QAXEB075) and the Electronics
Engineering Research DIvision of the Lawrence LIvermore Laboratory for their support of the work reported here.

NOTICE

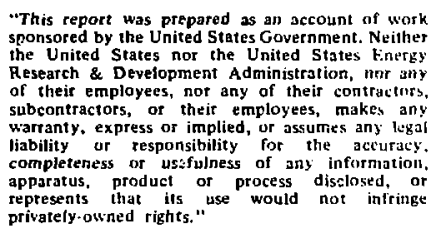

